\title{
Fiebre de origen desconocido en niños: experiencia de 5 años en un hospital pediátrico de Perú
}

\section{Fever of unknown origin in children: a 5-year experience in a pediatric hospital in Peru}

\author{
Silvia Cerdán-Rojas, * Jorge Candela-Herrera, ${ }^{*}$ \\ Kevin Flores-Lovon, ${ }^{\ddagger}$ Ericson L Gutiérrez-Ingunza ${ }^{\S}$ \\ * Instituto Nacional de Salud del Niño, Lima, Perú; ${ }^{\ddagger}$ Universidad Nacional de San Agustín de Arequipa. Arequipa, Perú; \\ $\S$ Universidad San Ignacio de Loyola, Unidad de Investigación para la Generación y Síntesis de Evidencias en Salud, Lima, Perú.
}

\section{RESUMEN}

Objetivos: Describir las características clínicas y epidemiológicas de los niños con fiebre de origen desconocido (FOD) en el Instituto Nacional de Salud del Niño de Lima, Perú. Material y métodos: Estudio descriptivo y retrospectivo con 100 pacientes menores de 17 años, hospitalizados entre los años 2006 y 2010. FOD se basó en los siguientes criterios: fiebre $>38.3^{\circ} \mathrm{C}$, con una evolución $\geq 3$ semanas, sin causa establecida, o bien, paciente febril sin diagnóstico etiológico después de tres días de hospitalización. Se revisaron expedientes clínicos, obteniéndose datos de interrogatorio, exploración física y de hallazgos de laboratorio y gabinete. Resultados: Del total, $49 \%$ tenía menos de dos años. Alguna enfermedad infecciosa fue la causa más frecuente de FOD (48\%), seguido de neoplasias (6\%), y $2 \%$ por otras causas. En $44 \%$ no se llegó a un diagnóstico específico. La enfermedad por arañazo de gato (8\%) fue la principal etiología infecciosa, seguido de infección del tracto urinario (7\%). En cuanto a las neoplasias, cinco pacientes tuvieron leucemia y uno histiocitosis. Conclusiones: En pacientes pediátricos con FOD, la etiología infecciosa fue la causa más frecuente, seguida de una enfermedad neoplásica. Existe un porcentaje elevado de pacientes con FOD en quienes no se llega a determinar su etiología.

Palabras clave: Fiebre de origen desconocido, niño, enfermedades infecciosas, Perú.

\begin{abstract}
Objectives: To describe the clinical and epidemiological characteristics of children with fever of unknown origin (FUO) at the National Institute of Child Health in Lima, Peru. Material and methods: Descriptive and retrospective study with 100 patients younger than 17 years of age, hospitalized between 2006 and 2010. FUO was based on the following criteria: fever $>38.3^{\circ} \mathrm{C}$, with an evolution $\geq 3$ weeks, without established cause, or febrile patient without diagnosis etiology after three days of hospitalization. Clinical records were reviewed, obtaining personal history and physical examination data, as well as laboratory and imaging findings. Results: Of the total, $49 \%$ were under two years of age. An infectious disease was the most frequent cause of FUO (48\%), followed by neoplasms (6\%), and $2 \%$ due to other causes. In $44 \%$ no specific diagnosis was reached. Cat scratch disease (8\%) was the main infectious etiology, followed by urinary tract infection (7\%). Regarding neoplasms, five patients had leukemia and one histiocytosis. Conclusions: In pediatric patients with FUO, infectious aetiology was the most frequent cause, followed by neoplastic disease. There is a high percentage of patients with FUO in whom its etiology cannot be determined.
\end{abstract}

Keywords: Fever of unknown origin, child, infectious diseases, Peru.

Correspondencia: Silvia Cerdán-Rojas, E-mail: scerdanrojas@gmail.com

Citar como: Cerdán-Rojas S, Candela-Herrera J, Flores-Lovon K, Gutiérrez-Ingunza EL. Fiebre de origen desconocido en niños: experiencia de 5 años en un hospital pediátrico de Perú. Rev Mex Pediatr. 2021; 88(5): 179-183. https://dx.doi.org/10.35366/103896 


\section{INTRODUCCIÓN}

La fiebre se define como la elevación de la temperatura por encima de la variación diaria normal, es uno de los motivos de consulta más frecuentes en pediatría, especialmente entre los 3-36 meses de vida, y hasta en $65 \%$ de los menores de dos años corresponde al principal motivo de consulta. ${ }^{1}$

La mayoría de los niños con fiebre o síndrome febril presentan enfermedades cuya causa es relativamente sencilla de identificar y dar tratamiento específico. Sin embargo, a pesar del escrutinio, existen pacientes en quienes la fiebre persiste por más de una semana, sin encontrar su etología. Este grupo corresponde a lo que se denomina fiebre de origen desconocido (FOD), en el cual se debe establecer un orden para el abordaje de las posibles causas. ${ }^{2}$

La FOD representa 0.5-3\% de las hospitalizaciones pediátricas. ${ }^{3}$ A pesar de la aparición de nuevas pruebas para identificar la causa, no se ha logrado observar cambios significativos en el proceso diagnóstico de FOD en los últimos años. ${ }^{4}$ La búsqueda de la causa genera un gasto considerable en hospitalización pero, además, existe un porcentaje de pacientes en quienes no se logra establecer el diagnóstico. ${ }^{5}$

El abordaje de niños con FOD incluye un interrogatorio y examen físico dirigidos hacia las posibles causas, además de la solicitud de estudios de laboratorio y radiológicos complementarios. ${ }^{6,7}$ En general, la secuencia de la solicitud de estos exámenes se basa en la frecuencia de la etiología en el lugar de origen de los pacientes. ${ }^{8}$

En el ámbito internacional, 51\% de las causas de FOD tienen origen infeccioso, 25\% no infeccioso, como las enfermedades reumatológicas o neoplásicas y en aproximadamente $23 \%$ no se llega a un diagnóstico. ${ }^{9}$ Esta frecuencia es similar a lo realizado en Perú, en el año $2004 .^{10}$

En el presente estudio se describen las características clínicas y epidemiológicas de los niños con FOD en el servicio de Infectología del Instituto Nacional de Salud (INSN) del Niño de Lima, Perú.

\section{MATERIAL Y MÉTODOS}

Estudio descriptivo, transversal y retrospectivo, en el que se incluyeron pacientes de un mes a 17 años hospitalizados entre enero de 2006 y diciembre de 2010, con el diagnóstico de FOD. Se excluyeron pacientes que tenían una enfermedad de base conocida, como enfermedad cardiovascular, hepática, pulmo- nar, gastrointestinal, endocrinológica, metabólica, entre otras.

El INSN es una institución nivel III-2 según la normativa peruana. Este hospital es considerado de alta complejidad, en el cual se atienden principalmente niños que cuenten con el Seguro Integral de Salud, para personas de bajos recursos económicos. En su mayoría, el hospital recibe pacientes provenientes de otros hospitales de menor complejidad tanto de la ciudad de Lima, como de provincias.

Para considerar la FOD, se tomó en cuenta: fiebre mayor de $38.3{ }^{\circ} \mathrm{C}$ documentada en varias

Tabla 1: Características clínicas y epidemiológicas de 100 pacientes con fiebre de origen desconocido.

Características $n(\%)$

Sexo

Hombre 55 (55)

Mujer $\quad 45(45)$

Edad

0-1 $49(49)$

$2-4 \quad 20(20)$

$5-9$

$10-15$

Procedencia

Urbano $50(50)$

Rural $\quad 50(50)$

Viajes

Costa $11(11)$

Sierra 7 (7)

Selva 2 (2)

Animales

Perro 44 (44)

Gato 21 (21)

Aves 24 (24)

Roedores (cuyos) 15 (15)

Cerdos 2 (2)

Contacto tuberculosis

Sí

$16(16)$

Características clínicas

Disminución de peso 54 (54)

Sudoración 36 (36)

Adenopatías 27 (27)

Visceromegalias 21 (21)

Palidez 9 (9)

Dolor abdominal $3(3)$

Ictericia 2 (2)

Días de hospitalización

Mediana [rango] $\quad 10$ [3-64] 
Tabla 2: Diagnóstico final de 100 pacientes pediátricos con fiebre de origen desconocido.

$\begin{array}{ll}\text { Diagnósticos } & \text { n } \\ \text { Sin diagnóstico } & 44 \\ \text { Arañazo de gato } & 8 \\ \text { Infección del tracto urinario } & 7 \\ \text { Infección por citomegalovirus } & 5 \\ \text { Leucemia } & 5 \\ \text { Tifoidea } & 5 \\ \text { Sinusitis } & 4 \\ \text { Neumonía adquirida en la comunidad } & 3 \\ \text { Otitis media aguda } & 3 \\ \text { Brucelosis } & 2 \\ \text { Tuberculosis pulmonar } & 2 \\ \text { Absceso dentario } & 1 \\ \text { Infección por virus de Epstein Barr } & 1 \\ \text { Fasciolasis } & 1 \\ \text { Hipereosinofilia } & 1 \\ \text { Histiocitosis } & 1 \\ \text { Otitis media crónica } & 1 \\ \text { Osteomielitis } & 1 \\ \text { Paracoccidioidomicosis diseminada } & 1 \\ \text { Quiste hidatídico abscedado } & 1 \\ \text { Meningoencefalitis tuberculosa } & 1 \\ \text { Toxocariasis } & 1 \\ \text { Trombosis venosa profunda } & \end{array}$

ocasiones, duración de la fiebre por más de tres semanas y que el diagnóstico etiológico permaneciera desconocido después de una semana de estudio hospitalario. ${ }^{11,12}$

El protocolo de los niños con FOD incluye la realización de los siguientes exámenes de laboratorio: hemograma completo, velocidad de sedimentación globular (VSG), proteína $\mathrm{C}$ reactiva (PCR), transaminasas (AST, ALT), fosfatasa alcalina, deshidrogenasa láctica (LDH), creatinfosfocinasa (CPK). Además de examen de orina, aglutinaciones para Brucella y Salmonella, serología (IgM/IgG) para toxoplasma, rubeola, citomegalovirus, herpes, Bartonella henselae. Asimismo, hemocultivo, urocultivo y búsqueda de bacilo de Koch (BK) en esputo/aspirado gástrico para la identificación de agentes microbianos.

En cuanto a estudios de gabinete, se solicita Rx de tórax y de senos paranasales, así como ecografía abdominal, al igual que evaluación por servicio de odontología.

Para este estudio, se revisaron expedientes clínicos para obtener los datos demográficos y clí- nicos, los cuales se incluyeron en una base de datos electrónica.

El análisis estadístico fue de tipo descriptivo, los datos se presentan con frecuencias y porcentajes, así como mediana y valores mínimo y máximo. Se empleó el programa SPSS versión 22.0.

\section{RESULTADOS}

Se incluyeron 100 pacientes. En cuanto a la edad, el mayor porcentaje tenía menos de dos años (49\%); $55 \%$ fueron varones, $50 \%$ provenían del ámbito rural. Del total, $20 \%$ tenían antecedente de algún viaje antes de iniciar con fiebre, $11 \%$ a la costa, $11 \%$ a la sierra y $7 \%$ a la selva. Además, $44 \%$ de los pacientes tenían animales domésticos, principalmente perros ( $44 \%$ ) y gatos $(21 \%)$, pero $24 \%$ criaban aves, y $15 \%$ señalaron que convivían con roedores y $2 \%$ con cerdos (Tabla 1).

La mediana de días con fiebre antes del ingreso al hospital fue de 30 días (rango 22-90 días). Además de la fiebre, $54 \%$ de los pacientes refirieron disminución de peso, y 36\% sudoración. De la exploración física (EF), en $27 \%$ se identificaron adenopatías, $21 \%$ con hepatoesplenogalia y palidez en $9 \%$.

De acuerdo con el protocolo señalado, el diagnóstico final se logró en 56 pacientes, de los cuales en 48 la fiebre de atribuyó a un proceso infeccioso, en seis a una neoplasia, uno tuvo eosinofilia y el último trombosis venosa. Por lo anterior, en $44 \%$ no se llegó a diagnóstico nosológico definido. En la Tabla 2 se especifican los diagnósticos etiológicos; como se muestra, la enfermedad por arañazo de gato (8\%) fue lo más frecuente, seguido por la infección de tracto urinario (7\%).

Tabla 3: Diagnóstico de egreso por grupos etarios en pacientes con diagnóstico de fiebre de origen desconocido.

Diagnóstico, $\mathrm{n}(\%)$

Edad Infeccioso Neoplásico Otros Sin diagnóstico Total

$\begin{array}{lcllcr}0-2 & 18(36.7) & 2(4.1) & 1(2.0) & 28(57.1) & 49 \\ 2-5 & 14(70.0) & 1(5.0) & 0(0.0) & 5(25.0) & 20 \\ 5-10 & 12(66.7) & 1(5.6) & 0(0.0) & 5(27.8) & 18 \\ 10-15 & 4(33.3) & 2(16.7) & 1(8.3) & 5(41.7) & 12 \\ >15 & 0(0.0) & 0(0.0) & 0(0.0) & 1(100.0) & 1 \\ \text { Total } & 48(48.0) & 6(6.0) & 2(2.0) & 44(44.0) & 100\end{array}$


Tabla 4: Hallazgos de laboratorio* en 100 pacientes pediátricos con fiebre de origen desconocido.

\begin{tabular}{|c|c|c|c|c|}
\hline \multirow[b]{2}{*}{$\begin{array}{l}\text { Examen de } \\
\text { laboratorio }\end{array}$} & \multicolumn{4}{|c|}{ Diagnóstico final } \\
\hline & $\begin{array}{c}\text { Infeccioso } \\
\mathrm{N}=48\end{array}$ & $\begin{array}{l}\text { Neoplásico } \\
\qquad N=6\end{array}$ & $\begin{array}{l}\text { Otros } \\
N=2\end{array}$ & $\begin{array}{l}\text { Sin diagnóstico } \\
\qquad N=44\end{array}$ \\
\hline Leucocitos (cel/mm³) & $10,820(5,000-20,200)$ & $8,100(1,300-45,000)$ & $12,295(8,400-16,190)$ & $9,540(4,000-15,900)$ \\
\hline Hemoglobina (mg/dL) & $9.90(5-13)$ & $8.7(5-15)$ & $8.25(7-10)$ & $10.50(7-11)$ \\
\hline Plaquetas (cel/mm³) & $431,000(34,600-800,000)$ & $373,000(22,000-600,000)$ & $312,000(303,000-321,000)$ & $400,000(56,000-799,000)$ \\
\hline PCR (mg/L) & $3.85(4-132)$ & $8.05(2-22)$ & $13.00(2-24)$ & $0.10(0-19)$ \\
\hline VSG $(\mathrm{mm} / \mathrm{h})$ & $50.00(9-72)$ & $57.00(50-65)$ & & $30(3-70)$ \\
\hline Ferritina (ng/mL) & $62.57(4-544)$ & $401.92(122-682)$ & & $51.01(2-662)$ \\
\hline
\end{tabular}

*Valores expresados en mediana (mínimo-máximo).

\section{Forma de llegar al diagnóstico etiológico}

Los agentes identificados en las infecciones del tracto urinario fueron: E. coli (cuatro casos), Klebsiella sp., Pseudomonas aeuruginosa y Citrobacter sp. El diagnóstico de fiebre tifoidea se realizó por la detección de aglutinaciones para Salmonella y hemocultivo positivo para Salmonella typhi. El diagnóstico de brucelosis se realizó por el método de aglutinaciones, rosa de bengala y prueba en placa. Los casos de sinusitis fueron identificados por tomografía computarizada; para tuberculosis fue mediante aislamiento del BK en esputo y/o aspirado gástrico, o en líquido cefalorraquídeo.

En los casos de neumonía, el diagnóstico se realizó por hallazgos radiográficos, mientras que otitis por EF. Toxocariasis fue por anticuerpos IgG, y fasciolasis por prueba positiva doble difusión arco 2 .

El niño con paracoccidiomicosis diseminada se detectó por biopsia de colon, donde se observó Paracoccidioides brasiliensis. El caso de quiste hidatídico fue identificado por estudios de imagen y visualización del agente después de quistectomía.

En cuanto a las enfermedades oncológicas, el diagnóstico se llegó por biopsia de médula ósea en los cinco pacientes con leucemia; mientras que el paciente con histiocitosis fue por biopsia de ganglio cervical.

\section{Factores relacionados con FOD}

A fin de señalar algunos datos que pudieran orientar a la etiología de FOD se presentan las Tablas 3 y 4 . En la primera, se puede observar que la mayoría de los diagnósticos de origen infeccioso ocurren en niños menores de dos años, pero también en este mismo grupo de edad se concentra la mayoría de los casos sin diagnóstico.

En cuanto a los hallazgos de laboratorio (Tabla 4), apreciamos que la mediana de los leucocitos fue de $10,200 / \mathrm{mm}^{3}$, además de la mayoría de niveles bajos de hemoglobina (mediana de $10 \mathrm{mg} / \mathrm{dL}$ ). La mediana del valor de plaquetas fue de $400,000 / \mathrm{mm}^{3}$, de la proteína $\mathrm{C}$ reactiva (PCR) fue de $1.9 \mathrm{mg} / \mathrm{L}$, de la velocidad de sedimentación globular (VSG) fue $41 \mathrm{~mm} / \mathrm{h}$ y de la ferritina $59 \mathrm{ng} / \mathrm{mL}$.

\section{Evolución de pacientes con FOD}

En 41 pacientes, la fiebre remitió con tratamiento empírico de acuerdo con la sospecha clínica del cuadro clínico, y en 11 con tratamiento dirigido al comprobar el diagnóstico. En 36 casos, la fiebre se autolimitó. Seis pacientes fueron derivados al Instituto Nacional de Enfermedades Neoplásicas y, por último, hubo seis pacientes en quienes la fiebre no remitió.

Por otro lado, en cuanto a los 44 pacientes en quienes no se estableció diagnóstico definitivo, en 73\% (32) la fiebre remitió durante la hospitalización, y en seis (14\%) remitió con tratamiento empírico. Por último, seis pacientes mayores de 10 años persistieron con fiebre, pero se decidió su egreso, porque no se encontró algún síntoma extra además de la fiebre. Sin embargo, fueron seguidos de manera ambulatoria, verificando que la fiebre remitió de manera espontánea.

Finalmente, señalamos que 10 días fue la mediana de hospitalización (rango 3-64 días). Mientras que entre quienes la fiebre se autolimitó, el valor fue de nueve días (rango 2-20 días). 


\section{DISCUSIÓN}

En la presente investigación encontramos que la principal causa de FOD en la población estudiada fue de origen infeccioso y un menor porcentaje neoplásico; sin embargo, en un alto porcentaje no se llegó a un diagnóstico. Estudios previos muestran también que la principal causa es de origen infeccioso, aunque la segunda causa en frecuencia tiene una gran variabilidad, incluyendo enfermedades neoplásicas, autoinmunes y colagenopatías. ${ }^{13-15}$

En Corea del Sur se reportó que el porcentaje de niños en los que no se llegó a un diagnóstico fue de $43 \%$, resultado similar al presente estudio. ${ }^{16}$ Sin embargo, en otras series, el porcentaje de casos sin un diagnóstico final es menor. ${ }^{13-15}$

Otro punto importante es que, en algunos casos, la etiología del FOD podría haberse identificado en establecimientos de menor nivel de complejidad, como las infecciones urinarias o fiebre tifoidea. Lamentablemente, el proceso de referencia y contrarreferencia entre instituciones de diferentes niveles de atención no está bien establecido, lo que lleva a los pacientes a acudir directamente a hospitales de tercer nivel, sobre todo si los niños llevan varios días con fiebre.

En este estudio, la etiología más frecuente de FOD fue la enfermedad por arañazo de gato, la cual es una enfermedad infecciosa aguda, por lo general benigna producida por Bartonella henselae. Esta enfermedad es una causa frecuente de inflamación ganglionar crónica, tiene una evolución, por lo general, autolimitada, pero también se han descrito casos que solamente tienen fiebre. ${ }^{17}$ Otros estudios en Perú también ubican a esta enfermedad como la principal causa de FOD en niños. ${ }^{18}$

La segunda causa de origen de FOD fueron las infecciones del tracto urinario (7\%), resultados muy parecidos a los encontrados en un hospital pediátrico en Papúa, Nueva Guinea (9\%). ${ }^{19}$ Por lo que parece importante siempre incluir los urocultivos como parte del proceso diagnóstico en niños con fiebre sin foco aparente. ${ }^{20}$

Una de las limitaciones del presente estudio fue que se trata de un solo centro hospitalario, por lo que podría no ser representativo de toda la población de niños con FOD.

\section{REFERENCIAS}

1. Ares J, Morillo B. Manejo del paciente pediátrico con fiebre sin foco. En: AEPap (ed). Congreso de Actualización Pediatría 2020. Madrid: Lúa Ediciones 3.0; 2020: 397-408.

2. Mendez A, Velazquez R. Fiebre de origen desconocido. An Pediatr Contin. 2009; 7(4): 205-213.

3. Chow A, Robinson JL. Fever of unknown origin in children: A systematic review. World J Pediatr. 2011; 7(1): 5-10.
4. Cho C-Y, Lai C-C, Lee M-L, Hsu C-L, Chen C-J, Chang L-Y et al. Clinical analysis of fever of unknown origin in children: a 10year experience in a northern Taiwan medical center. $J$ Microbiol Immunol Infect. 2017; 50(1): 40-45.

5. Szymanski AM, Clifford H, Ronis T. Fever of unknown origin: a retrospective review of pediatric patients from an urban, tertiary care center in Washington, DC. World J Pediatr. 2020; 16(2): 177-184.

6. Dayal R, Agarwal D. Fiebre en niños y fiebre de origen desconocido. Indian J Pediatr. 2016; 83: 38-43.

7. Chien YL, Huang FL, Huang CM, Chen PY. Clinical approach to fever of unknown origin in children. J Microbiol Immunol Infect. 2017; 50(6): 893-898.

8. Chouchane S, Chouchane $\mathrm{CH}$, Ben $\mathrm{MCH}$, Seket B, Hammami $\mathrm{S}$, Nouri $\mathrm{S}$ et al. Les fievres prolongées de l'enfant. Étude rétrospective de 67 cas. Arch Pediatr. 2004; 11: 1319-1325.

9. Barbi E, Marzuillo P, Neri E, Naviglio S, Krauss BS. Fever in children: pearls and pitfalls. Children (Basel). 2017; 4(9): 81.

10. Morales SCA, Shimabukuro KGY. Fiebre de origen desconocido en Niños, Servicio de Infectología del Instituto Especializado de Salud del Niño, 1995-2000. [Tesis] Trabajo de Investigación para optar el título de Especialista en Pediatría. Lima, 2004.

11. Petersdorí R, Beeson R. Fever on unexplained origin: report on 100 causes. Medicine. 1961; 40(1): 35-45.

12. Durack DT, Street AC. Fever of unknown origin re-examined and redefined. Curr Clin Top Infect Dis. 1991; 11: 35-51.

13. Flores TMU. Etiología de la fiebre de origen desconocido (FOD), pacientes pediátricos ingresados en el Departamento de Pediatría del Hospital Nacional Dos de Mayo, 2007-2012. [Tesis] Trabajo de Investigación para optar el título de Especialista en Pediatría. Lima, 2014.

14. Sandoval C, Pinochet C, PeñaA, Rabello M, Prado A, Viviani T. Fiebre de origen desconocido: un reto para el especialista en enfermedades infecciosas pediátricas. Rev Chil Infectol. 2014; 31(1): 87-91.

15. Yi-Seul K, Kyung-Ran K, Ji-Man Kang, Jong-Min K, Yae-Jean K. Etiology and clinical characteristics of fever of unknown origin in children: a 15-year experience in a single center. Korean J Pediatr. 2017; 60(3): 77-85.

16. Kim YS, Kim KR, Kang JM, Kim JM, Kim YJ. Etiology and clinical characteristics of fever of unknown origin in children: a 15-year experience in a single center. Korean J Pediatr. 2017; 60(3): 77-85.

17. Mori J, Salazar M, Salcedo E. Enfermedad por arañazo de gato como causa de fiebre de origen desconocido: reporte de caso. Rev Mex Pediatr. 2019; 86(3): 123-125.

18. Miranda-Choque E, Candela J, Alvarado J, Esquivel M, Farfán S. Infección por Bartonella henselae como presentación de fiebre de origen desconocida en niños peruanos. Acta Méd Peruana. 2014; 31(2): 74-78.

19. Morris CM, Tefuarani N, Ripa P, Laki R, Vince JD. Urinary tract infection in infants and young children presenting with fever without a focus in Port Moresby. P N G Med J. 2007; 50(3-4): 145-151.

20. Hamilton JL, John SP. Evaluation of fever in infants and young children. Am Fam Physician. 2013; 87(4): 254-260.

Aspectos éticos: El presente estudio se llevó a cabo respetando los principios éticos de la declaración de Helsinki. El estudio fue aprobado por el Comité de Ética en Investigación y registrado en el INSN. Toda la información se manejó de manera anónima y confidencial.

Conflicto de intereses: Los autores declaran que no tienen. 